Mathematical Modelling and Analysis

Volume 21 Number 6, November 2016, 762-773

http://dx.doi.org/10.3846/13926292.2016.1241835

(c) Vilnius Gediminas Technical University, 2016
Publisher: Taylor\&Francis and VGTU

http://www.tandfonline.com/TMMA

ISSN: 1392-6292

eISSN: $1648-3510$

\title{
Growth of a Gas Bubble in a Steady Diffusion Field in a Tissue Undergoing Decompression
}

\author{
Selim A. Mohammadein ${ }^{a}$ and Khaled G. Mohamed ${ }^{b}$ \\ ${ }^{a}$ Department of Mathematics, Faculty of Science, Tanta University \\ Tanta, Egypt \\ ${ }^{b}$ Department of Mathematics, Faculty of Science, Benha University \\ Benha, Egypt \\ E-mail(corresp.): khaled_goda@yahoo.com \\ E-mail: selimali2009@yahoo.com
}

Received July 6, 2015; revised September 21, 2016; published online November 15, 2016

\begin{abstract}
This paper presents parameterized study on the growth of a convective gas bubble in tissues of a steady diffusion field. Resulting formulae are obtained analytically, which are valid for constant ambient pressure. It's found that the growing bubble radius is proportional to initial bubble radius, initial concentration difference, diver's average temperature and initial void fraction which is the dominant parameter, while it's inversely proportional to surface tension, viscosity, density ratio and ambient pressure. Comparison to some previous work is performed.
\end{abstract}

Keywords: bubble growth, steady diffusion, tissue, concentration distribution, viscosity.

AMS Subject Classification: 76T10; 76A05; 76Zxx.

\section{Introduction}

Decompression sickness (DCS) is caused by the release of inert gas bubbles (usually nitrogen) into the bloodstream and tissues, after ambient pressure is reduced. At depth, the partial pressures of gasses in the breathing mixture increase in proportion to the ambient pressure, according to Dalton's law [14].

Although oxygen is actively metabolized, nitrogen is inert and becomes dissolved in body tissues until saturation that proportional to the ambient pressure as defined by Henry's law. The propensity for the formation of nitrogen bubbles depends on the depth of the dive, the length of time at depth and the rate of ascent. If ambient pressure is released too quickly, the dissolved nitrogen gas that cannot remain in solution will form air bubbles within the blood, interstitial fluids and organs [14].

The physiological problems associated with decompression from elevated atmospheric pressures have been known for over 100 years. These problems can generally be divided into two broad categories: 1) those due to physical 
injury as a result of an expansion of gas and 2) those due to a liberation of a gas phase in tissues [13]. Figure 1 demonstrates how overexpanding gas ruptures alveoli and is forced into the pulmonary vasculature. Gas is then distributed systemically, causing arterial gas embolism $[12,13]$.

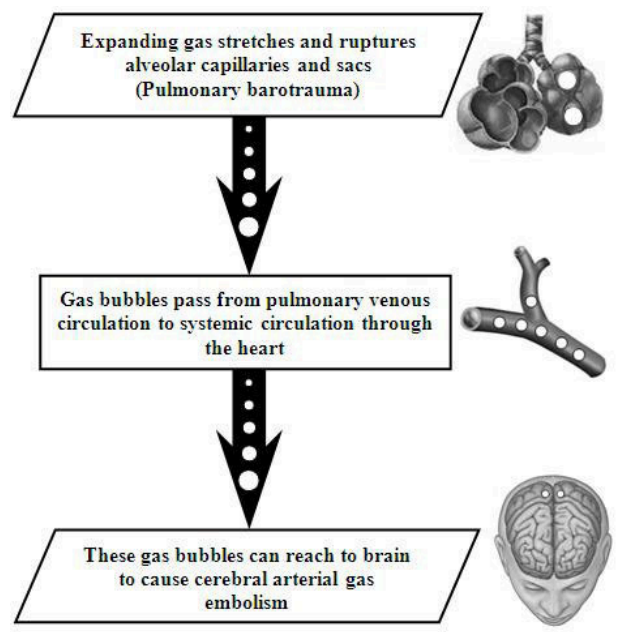

Figure 1. This algorithm shows how overexpanding gas ruptures alveoli and produce air bubbles, which can be distributed through systemic circulation to other body tissues causing, sometimes, arterial gas embolism.

As well as this bubbles appear in tissues of divers who surface too quickly it can also do in people who flight for long distances from the earth [11].

Bubble dynamics models suitable for these applications assume the bubble to be either contained in an unstirred tissue (two-region model) in which gas exchange between bubble and tissue is limited by bulk diffusion through the tissue $[5,8,20,21]$, or surrounded by a boundary layer within a well-stirred tissue (three-region model) in which diffusion-limited exchange of gas between bubble and tissue occurs through a boundary layer surrounding the growing gas bubble $[4,10,11,18,20,21]$. More literature about previous models that describing the growth of gas bubbles in tissues and blood can be found in $[2,15,18,20]$.

R.S. Srinivasan et al. $[20,21]$ used three-region model to solve the growth problem in the case of quasi-static pressure in two cases, the first was under assumption of fixed thickness of the boundary layer [20], the other was under assumption of variable thickness of the boundary layer [21] . Mohammadein and Mohamed [11] solved the problem in unsteady case regardless the thickness of the boundary layer.

In this work, we solved the problem analytically for the case of steady gas diffusion (quasi-static pressure) from the supersaturated gas tension in the tissue into an expanding gas bubble under effect of convective acceleration SCD model. Several authors apply their model formula to the entire growth period $[1,4,18,19,20,21]$, which is not accurate, because the growth period can be divided into consecutive steps [11] (see Figure 3), some of these steps are 
at which the ambient pressure is decreased, while the diver is ascending too quickly, and other growth steps are at which the ambient pressure is constant at diving steps or at the sea level after complete ascending. The later step takes almost of the growth time, since the diver can ascend to the sea level in some seconds or few minutes but his tissues still supersaturated and the growth process is still taking place. This model is valid to describe the growth stages at which the ambient pressure is constant, these periods may last for many minutes or some hours as predicted by many of previous models. The effect of bubble convection, which really affects on the growth process as we show in this study, was omitted by several authors like $[1,4,5,8,20,21]$. The effect of the body fluids viscosity is also included in this study, in contrary of previous studies that omitted it like $[1,2,5,8,9,10,11,20,22]$.

The solution is valid for the case of constant ambient pressure(through diving stops or at sea level or any altitude of constant ambient pressure). Moreover, a formula for concentration distribution around a growing gas bubble is also derived. The results are implemented to explain the effects of the given physical parameters on the growth of a gas bubble in a diver's tissue, who surface quickly to the sea level.

\section{Analysis}

The three-region model (Gas bubble, uniform boundary layer and well-stirred finite tissue "tissue compartment") is used. For simplicity, solvent (tissue fluid) vapor pressure is neglected and a single diffusible gas cases is involving only.

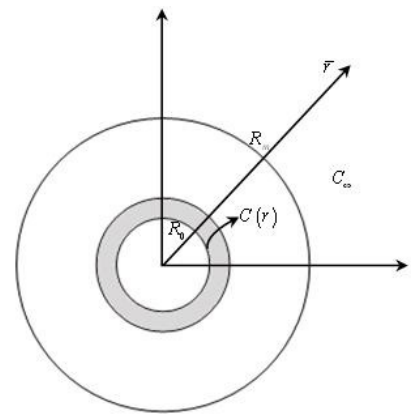

Figure 2. Problem Sketch.

As shown in Figure 2, a single gas bubble is considered to grow inside a finite tissue between two finite radius boundaries $R_{0}$ and $R_{m}$, the growth is affected by surrounding physical parameters such as the pressure difference $\Delta P$ between the bubble pressure $P_{g}(R(t), t)$ and the ambient pressure $P_{a m b}$, surface tension of the mixture inside the tissue at the bubble boundary, tissue fluid viscosity, concentration difference between the two phases and other physical parameters as will be shown through the text.

The following assumptions are taken into account: 
- Diffusible gas is considered to be ideal.

- The bubble is assumed to have a spherical geometry, which is valid in microscopic scale.

- Pressure inside the bubble is assumed to be uniform.

- Gas density distribution inside the bubble is assumed to be uniform except for a thin boundary layer near the bubble wall.

- The solvable gas tension in the tissue outside the boundary layer is uniform and has slight variations with time $\frac{\partial C}{\partial t} \rightarrow 0$ (quasi-static approximation) [20].

This approximation can be considered by assuming that the loss in diffusible gas tension by diffusion to the growing gas bubble (sink) can be replaced by perfusion from blood to tissue fluid. The validity of this approximation may not hold during very rapid changes in ambient pressure or breathing gas. In such cases, changes in bubble radius may have to be determined by using the complete diffusion equation [20].

The mathematical model describing this problem consists of four main equations: mass balance, diffusion, Fick's and Laplace equations.

\section{Mass balance equation}

This equation determines tissue gas tension. Assuming equilibration of tissue gas with venous blood gas. The rate of gas uptake by the tissue is the amount carried by the blood per unit time less the flux into the gas bubble. Thus, the mass equation has the form [20]

$$
\alpha_{T} V_{T} \frac{d P_{T}}{d t}=\alpha_{b} V_{T} \dot{Q}\left(P_{a}-P_{T}\right)-\frac{1}{\Re T} \frac{d}{d t}\left(P_{g} V_{g}\right) .
$$

\section{Diffusion equation}

This equation describes diffusion of gas through tissue. Steady convective mass equation without any source or sink, under assumption of quasistatic approximation $[6,20,23]$, and assuming spherical symmetry is

$$
\frac{\varepsilon R^{2} \dot{R}}{r^{2}} \frac{\partial C}{\partial r}=D_{T}\left(\frac{\partial^{2} C}{\partial r^{2}}+\frac{2}{r} \frac{\partial C}{\partial r}\right),
$$

where $\varepsilon$ is the density ratio that defined as $\varepsilon=1-\rho_{g} / \rho_{l}[17]$.

\section{Fick's equation}

This equation allows calculation of the gas flux through the bubble surface. The rate of change of molar concentration of gas in the bubble equals the molar flux of gas through the bubble surface. Thus $[5,20]$

$$
\frac{1}{\Re T} \frac{d}{d t}\left(\frac{4}{3} \pi R^{3} P_{g}\right)=4 \pi R^{2} D_{T}\left(\frac{\partial C}{\partial r}\right)_{r=R} .
$$




\section{Pressure balance equation}

This equation describes the pressure balance at the bubble interface. Taking into account the effect of surface tension at the gas-liquid interface, and tissue fluid dynamic viscosity, the Laplace's equation that represents the pressure balance on the gas-liquid interface is $[3,16]$

$$
P_{g}=P_{a m b}+\frac{2 \sigma}{R}+\frac{4 \eta}{R} \dot{R}
$$

The general solution of Equation (2.1) is

$$
C(r)=k_{1} \frac{D_{T}}{\varepsilon R^{2} \dot{R}} e^{-\frac{\varepsilon R^{2} \dot{R}}{D_{T}^{r}}}+k_{2} .
$$

To find the constants $k_{1}$ and $k_{2}$, we apply the following boundary conditions:

$$
\text { At } r=R \Rightarrow C(r)=C_{R} \text { and at } R=R_{m} \Rightarrow C(r)=C_{\infty} .
$$

Then $k_{1}$ and $k_{2}$ can be expressed in the following form:

$$
\begin{aligned}
& k_{1}=\varepsilon R^{2} \dot{R}\left(C_{\infty}-C_{R}\right) / D_{T}\left(e^{-\frac{\varepsilon R^{2} \dot{R}}{D_{T} R}}-e^{-\frac{\varepsilon R \dot{R}}{D T}}\right), \\
& k_{2}=\left(C_{\infty} e^{\frac{\varepsilon R^{2} \dot{R}}{D_{T} R_{m}}}-C_{R} e^{\frac{\varepsilon R \dot{R}}{D_{T}}}\right) /\left(e^{\frac{\varepsilon R^{2} \dot{R}}{D_{T} R_{m}}}-e^{\frac{\varepsilon R \dot{R}}{D_{T}}}\right) .
\end{aligned}
$$

Therefore, the general solution (2.4) will be

$$
\begin{gathered}
C(r)=\frac{e^{-\frac{\varepsilon R \dot{R}}{D_{T}} \frac{R}{r}}\left(C_{\infty} e^{\frac{\varepsilon R \dot{R}}{D_{T}} \frac{R}{R_{m}}}\left(e^{\frac{\varepsilon R \dot{R}}{D_{T}}}-e^{\frac{\varepsilon R \dot{R}}{D_{T}} \frac{R}{r}}\right)\right.}{e^{\frac{\varepsilon R \dot{R}}{D_{T}}}-e^{\frac{\varepsilon R \dot{R}}{D_{T}} \frac{R}{R_{m}}}} \\
+\frac{\left.C_{R} e^{\frac{\varepsilon R \dot{R}}{D_{T}}}\left(e^{\frac{\varepsilon R \dot{R}}{D_{T}} \frac{R}{r}}-e^{\frac{\varepsilon R \dot{R}}{D_{T}} \frac{R}{R_{m}}}\right)\right)}{e^{\frac{\varepsilon R \dot{R}}{D_{T}}}-e^{\frac{\varepsilon R \dot{R}}{D_{T}} \frac{R}{R_{m}}}} .
\end{gathered}
$$

Therefore,

$$
\frac{\partial C}{\partial r}=-\frac{\varepsilon R^{2} \dot{R}\left(C_{\infty}-C_{R}\right)}{D_{T}\left(e^{\frac{\varepsilon R \dot{R}}{D_{T}} \frac{R}{R m}}-e^{\frac{\varepsilon R \dot{R}}{D_{T}}}\right) r^{2}} e^{\frac{\varepsilon R \dot{R}}{D_{T}}\left(1-\frac{R}{r}+\frac{R}{R_{m}}\right)}
$$

The boundary condition (2.2), by using of equation (2.3), is modified to be

$$
\left.\frac{\partial C}{\partial r}\right|_{r=R}=\frac{\left(4 \sigma+8 \eta+3 P_{a m b} R\right) \dot{R}+4 \eta R \ddot{R}}{3 \Re T D_{T} R} .
$$

From equations (2.7) and (2.8)

$$
-3 \Re T \varepsilon R \dot{R}\left(C_{\infty}-C_{R}\right)=\left(\left(4 \sigma+8 \eta+3 P_{a m b} R\right) \dot{R}+4 \eta R \ddot{R}\right)\left(1-e^{\frac{\varepsilon R \dot{R}}{D_{T}}\left(1-\frac{R}{R_{m}}\right)}\right) .
$$

Next we put the radius formula in the following form [11]:

$$
R(t)=\sqrt{R_{0}^{2}+2 k D_{T}\left(t-t_{0}\right)}, \dot{R}(t)=k D_{T} / R(t), \ddot{R}(t)=-k^{2} D_{T}^{2} / R(t)^{3} .
$$


Then, using the initial values at $t=t_{0}$, yields

$$
\begin{aligned}
-3 \Re T \varepsilon k D_{T} R^{2} \dot{R}\left(C_{\infty}-C_{R}\right)= & ( \\
\times\left(1-e^{\varepsilon k\left(1-\varphi_{0}^{1 / 3}\right.}\right) &
\end{aligned}
$$

where $\varphi_{0}=\left(R_{0} / R_{m}\right)^{3}$ is the initial void fraction.

$$
\begin{gathered}
-3 \Re T \varepsilon \Delta C_{0} R_{0}^{2} D_{T} k \approx\left(\left(4 \sigma+8 \eta+3 P_{a m b 0} R_{0}\right) R_{0} D_{T} k-4 \eta D_{T}^{2} k^{2}\right) \\
\times\left(1-1-\varepsilon k\left(1-\varphi_{0}^{1 / 3}\right)-\frac{\varepsilon^{2} k^{2}}{2}\left(1-\varphi_{0}^{1 / 3}\right)^{2}\right),
\end{gathered}
$$

After omitting very small terms, we have

$$
k\left[\varepsilon A^{2} E k^{2}+2 A E k-F\right]=0,
$$

where

$$
A=1-\varphi_{0}^{1 / 3}, E=\left(4 \sigma+8 \eta+3 P_{a m b 0} R_{0}\right) R_{0} D_{T}, F=6 \Re T R_{0}^{2} \Delta C_{0} D_{T} .
$$

The values for $\mathrm{A}, \mathrm{E}$ and $\mathrm{F}$ are always positive quantities, thus equation (2.10) has three roots for $\mathrm{k}$, the first one is zero, which is rejected, the possible positive root of the other two roots, which is acceptable for bubble growth case, has the following expression

$$
k=(\sqrt{1+\varepsilon F / E}-1) / \varepsilon A .
$$

Substituting this value into equations (2.9), the instantaneous radius, and corresponding growth and acceleration rates can be calculated, from which we can get the following expression for the initial growth velocity:

$$
\dot{R}_{0}=k D_{T} / R_{0}
$$

The following is an equivalent expression for the instantaneous bubble radius

$$
R(t)=\sqrt{R_{0}^{2}+2 R_{0} \dot{R}_{0}\left(t-t_{0}\right)} .
$$

To implement this result for divers who surfaces too quickly to the sea level, we have two cases for the ambient pressure (see Figure 3). The first one of them occurs through decompression (while ascending to sea level) in which the ambient pressure varies with time. This case can be dealt with by any model that takes the variations of ambient pressure into account. The other one occurs at diving stops or after ascending to the sea level, in which the ambient pressure has a constant value, which can be dealt with the current model.

At diving stops or after reaching sea level, although the ambient pressure is constant, but the bubble growth still taking place because the tissue fluid is still supersaturated by the solvable gas. In this case, the ambient pressure equals the atmospheric pressure $\left(P_{a m b}=P_{a t m}=101.325 \mathrm{kPa}\right)$. 


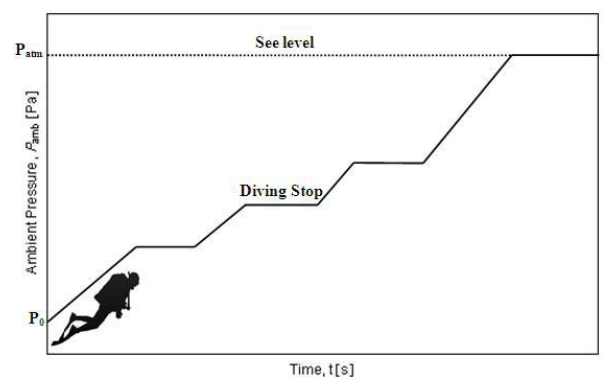

Figure 3. This figure shows the model validity at constant ambient pressure, which can be occurred at diving stops or at see level.

\section{Concentration distribution around a growing gas bub- ble in tissue}

From equations (2.9)

$$
R \dot{R}=k D_{T}
$$

Substituting into equations (2.6) it becomes

$$
C(r)=\frac{e^{-\varepsilon k R / r}\left(C_{\infty} e^{\varepsilon k R / R_{m}}\left(e^{\varepsilon k}-e^{\varepsilon k R / r}\right)+C_{R} e^{\varepsilon k}\left(e^{\varepsilon k R / r}-e^{\varepsilon k R / R_{m}}\right)\right)}{e^{\varepsilon k}-e^{\varepsilon k R / R_{m}}} .
$$

\section{Results and discussion}

Suppose a diver at some depth and stayed sufficient time for his blood and tissue fluids to be supersaturated with nitrogen. If he ascends quickly to the surface and didn't perform suitable regimes to washout the excess dissolved gas in his body, then the decompression of the surrounding ambient pressure will take place, hence nitrogen cavities release in vivo to constitute nitrogen microbubbles, which grow by consuming the excess amount of the dissolved gas by diffusion and it could be distributed systemically, causing arterial gas embolism and other symptoms of DCS may occurred. Although the decompression stage may last for some seconds or few minutes till the diver reaches sea level, the growth lasts for long time till full washout of the excess of the dissolved gas, this time may be many minutes or some hours as predicted by some of previous models $[11,20,21]$. The current model is implemented to this stage of constant ambient pressure at diving stops after decompression or at sea level or any altitude of constant ambient pressure.

The following Table 1 shows the data that used to simulate the problem by obtaining the following graphs that demonstrate the effect of some important physical parameters on the growth of the gas bubble in vivo.

The diffusion equation (2.1) represents the diffusion of supersaturated dissolved gas in tissue fluid into a growing gas bubble, under assumption of quasistatic approximation for dissolved gas tension. This equation is solved analyt- 
Table 1. Values of the constants and initial values of the parameters

\begin{tabular}{llll}
\hline & value & \multicolumn{2}{c}{ value } \\
\hline$R_{0}$ & $5.0 \times 10^{-6} \mathrm{~m}[20]$ & $T$ & $310.15\left(37^{\circ} \mathrm{C}\right) \mathrm{K}$ \\
$\Delta C_{0}$ & $0.7 \mathrm{~mol} / \mathrm{m}^{3}$ & $\Re$ & $8.314472 \mathrm{~N} . \mathrm{m} / \mathrm{mol} . K[7]$ \\
$\sigma$ & $0.03 \mathrm{~N} / \mathrm{m}[20]$ & $t_{0}$ & $0.0 \mathrm{~s}$ \\
$D_{T}$ & $2.2 \times 10^{-12} \mathrm{~m}^{2} / \mathrm{s}[20]$ & $\eta$ & $3.65 \times 10^{-3} \mathrm{Pa.s}$ \\
$\varepsilon$ & 0.99 & $\phi_{0}$ & $1.0 \times 10^{-3}[11]$ \\
\hline
\end{tabular}

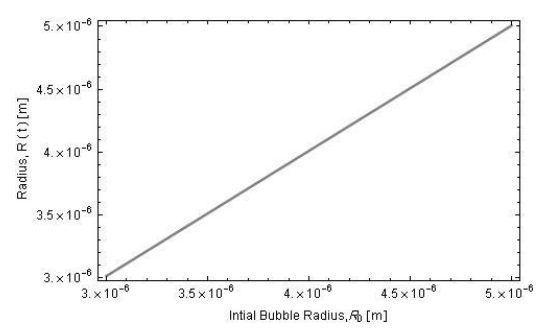

Figure 4. The relation between the bubble radius $R$ and the initial bubble radius $R_{0}$, for the range $3 \leq R_{0} \leq 5 \mu \mathrm{m}$.

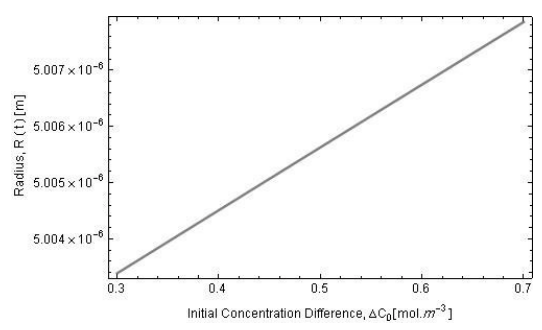

Figure 5. The relation between the bubble radius $R$ and the initial concentration difference $\Delta C_{0}$, for the range $0.3 \leq \Delta C_{0} \leq 0.7$ mol. $m^{-3}$.

ically in pair of the Fick's equation (2.2) and pressure balance equation (2.3) at the boundary conditions (2.5).

The problem solution represented by equations (2.9) and (2.11)-(2.14), gives explicitly the instantaneous bubble radius, growth rate and growth acceleration as a function of time combined with the surrounding physical parameters that affect on the growth process. Moreover, the concentration distribution around a growing gas bubble in tissue is presented by equation (3.1).

The time required for complete growth can be calculated by the following formula.

$$
t_{m}=\frac{R_{m}^{2}-R_{0}^{2}}{2 R_{0} \dot{R}_{0}}+t_{0} .
$$

By using the data listed in Table 1, Figures 4-11 explain the effect of changing the values of the parameters $R_{0}, \Delta C_{0}, T, \varphi_{0}, \sigma, \eta, \varepsilon$ and $P_{a m b}$ respectively on the bubble radius at some instant $(t=1.0 \mathrm{~s})$ through some intervals of these parameters. On the other hand, Figure 12 shows a comparison between the current model (Steady Convective Diffusion model SCD) and the previous models of Srinivasan et al [20] (Steady Non-Convective Diffusion model SNCD), and Mohammadein and Mohamed model [11] that solved the problem in the case of unsteady diffusion equation for a stationary growing gas bubble (USNCD model).

Figure 4 shows dependency of increasing the bubble growth rate on increasing of the initial bubble radius as well as it is found by the effect of increasing values of initial concentration difference (Figure 5), average body temperature (Figure 6) and initial void fraction (Figure 7). On contrary, Figure 8 shows dependency of decreasing the bubble growth rate on increasing of surface tension 


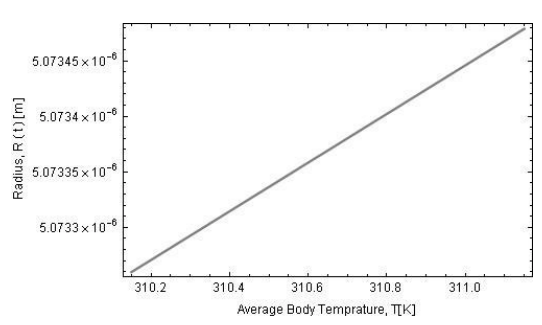

Figure 6. The relation between the bubble radius $R$ and the average body temprature

$T$, for the range $37^{\circ} \leq T \leq 38^{\circ} \mathrm{C}$.

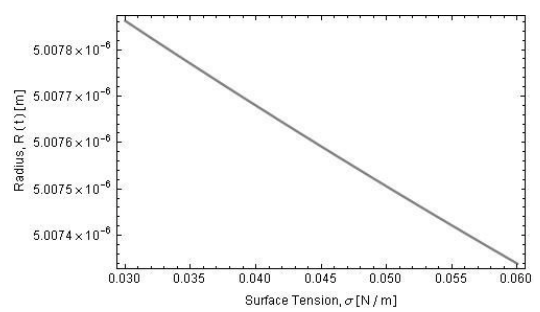

Figure 8. The relation between the bubble radius $R$ and the surface tension $\sigma$, through the range $0.03<\sigma<0.06 \mathrm{~N} . \mathrm{m}^{-1}$.

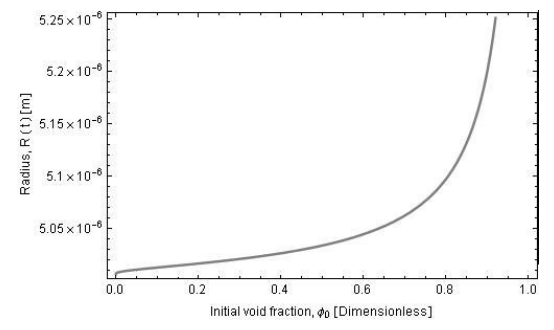

Figure 7. The relation between the bubble radius $R$ and the initial void fraction $\varphi_{0}$, through its range $0<\varphi_{0}<1$.



Figure 9. The relation between the bubble radius $R$ and the dynamic viscosity $\eta$, for the range $2 \times 10^{-3}<\eta<7 \times 10^{-3}$ Pa.s.

as well as it is found by the effect of increasing values of viscosity (Figure 9), density ratio (Figure 10) and surrounding ambient pressure (Figure 11), i. e., the growth of the gas bubble is proportional to the parameters $R_{0}, \Delta C_{0}, T$ and $\varphi_{0}$, while it's inversely proportional to the parameters $\sigma, \eta, \varepsilon$ and $P_{a m b}$. Moreover, the growth process is sensitive to slight changes in the initial void fraction at values near 1 , It can also be noticeable that the initial void fraction is the dominant parameter that affect on the growth process, next is the value of the ambient pressure.

The SNCD presented by Srinivasan et al. [20,21] of fixed boundary layer thickness predicted the time of growth to be about $82 \mathrm{~min}$. as shown in Figure 12, they also introduced a modified model of variable boundary layer thickness [21] that predicted the time of growth to be about $140 \mathrm{~min}$. On the other hand, the models that presented by the authors, the current SCD model and previous one USNCD model [11], although they depend also on the phenomena of thin boundary layer that adjacent to the bubble surface, but regardless its thickness. The USNCD model [11] predicts the time of growth to be about 50 min., while the SCD model predicts the time of growth to be about 363 min. . The initial growth rates for SCD model and USNCD model [11] are $3.15 \times 10^{-8} \mathrm{~m} . \mathrm{s}^{-1}$, and $2.65 \times 10^{-8} \mathrm{~m} . \mathrm{s}^{-1}$ respectively. This delay in the time of complete growth in SCD model may be due to the effect of dynamic viscosity that reduces growth rate, this parameter was omitted in the previous studies of Srinivasan et al [20,21] and Mohammadein and Mohamed [11].

Another notice is the growth rate can be sorted in the following ascending 


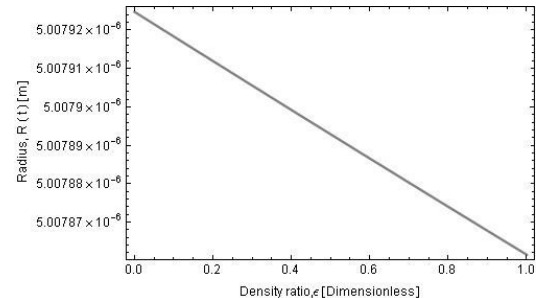

Figure 10. The relation between the bubble radius $R$ and density ratio $\varepsilon$, through its range $0<\varepsilon<1$.

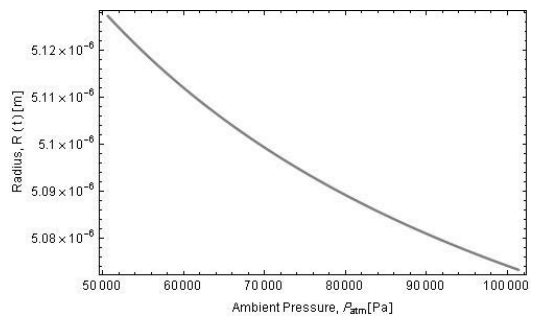

Figure 11. The relation between the bubble radius $R$ and the constant ambient pressure $P_{a m b}$, through the range $0.5<P_{a m b}<1 \mathrm{~atm}$.

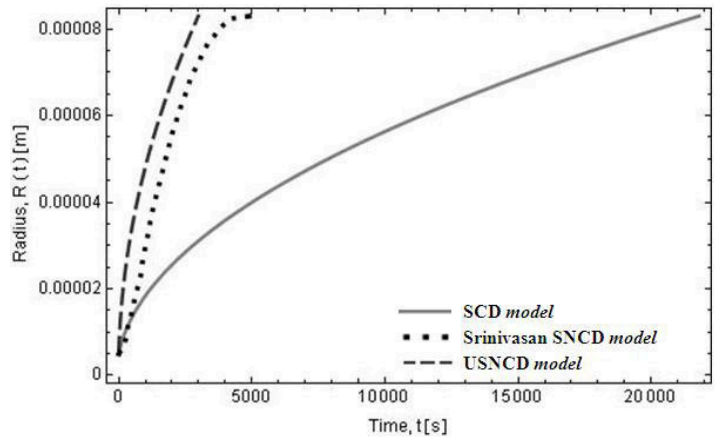

Figure 12. Comparison between the current model and previous models presented by Mohammadein and Mohamed [11] and Srinivasan et al [20,21] for $R_{0}=5 \mu \mathrm{m}, R_{m}=83 \mu \mathrm{m}$ and $\Delta C_{0}=3.0 \mathrm{~mol} . \mathrm{m}^{-3}$.

order; SCD model, SNCD model [20] and USNCD model [11]. That may explain the effect of consuming the excess in concentration of the supersaturated gas in the tissue by the growing bubble. That is, because in the Srinivasan et al model the perfusion of the nitrogen gas is considered, on the other hand in the USNCD model [11] the concentration of the dissolved gas changes with time is considered.

Figure 6 shows the effect of increasing of diver's average temperature on increasing of the bubble growth rate, which is the main reason of symptoms of DCS. It can also be shown from Figure 11 the risk of decreasing the ambient pressure that leads to increasing of the bubble growth rate, this explains why divers are advised to didn't flight directly after scuba diving, for example, taking a flight directly after scuba diving at altitude about $18000 \mathrm{ft}$ (at about half atmospheric pressure) leading to the risk of decompression sickness.

This model can be used in predicting the time that a growing gas bubble takes to reach a fixed radius $R_{m}$ by using of equation (4.1). This may be helpful in predicting the time that the bubble takes to reach some critical radius that can close some blood vessel, if its radius is known, which may cause an embolism. These results can also be used to avoid the harmful effects of 
DCS by reducing the bubble growth rates by suitable techniques by specialists to enforce the parameters that decrease the bubble growth rate and decrease the values of the parameters that increase the bubble growth rate.

Finally, we introduce the results of this study to specialized researchers to use it in applications of diving science like diving algorithms and diving tables, hoping that it is used in reducing the harmful effects of DCS, toward saving the divers health and life.

\section{Conclusions}

The growth of a gas bubble in an unperfused tissue in a steady diffusion field is discussed under effect of convective acceleration SCD model, based on the three-region model. The growing bubble radius is proportional to the parameters $R_{0}, \Delta C_{0}, T$ and $\varphi_{0}$, while it's inversely proportional to the parameters $\sigma, \eta, \varepsilon$ and $P_{a m b}$. The dominant parameter is the initial void fraction $\varphi_{0}$ that affects on the growth rate if a slight change in its value is performed near the value 1 , it also affects on the predicted initial growth rate. In addition, tissues of smaller viscosity values are predicted to have more bubble growth rates, leading to prediction of appearance of DCS symptoms first.

Moreover, the required time of complete growth, given by the formula (4.1), is greater than previous models $[11,20]$, in which the consuming of the supersaturated, dissolved gas in tissue and diffusion rates of it into the growing bubble is more than the presented model, the effect of viscosity in the current model is another reason of reducing the bubble growth rate.

\section{References}

[1] R. Ball, J. Himm, L.D. Homer and E.D. Thalmann. Does the time course of bubble evolution explain decompression decompression sickness risk? Undersea E Hyperbaric Medicine, 22(3):263-280, 1995.

[2] M.A. Chappell and S.J. Payne. A physiological model of the release of gas bubbles from crevices under decompression. Respiratory Physiology $\& 5$ Neurobiology, 153(2):166-180, 2006. http://dx.doi.org/10.1016/j.resp.2005.10.006.

[3] J.B. Freund. Suppression of shocked-bubble expansion due to tissue confinement with application to shock-wave lithotripsy. The Journal of the Acoustical Society of America, 123(5):2867-2874, 2008. http://dx.doi.org/10.1121/1.2902171.

[4] M.L. Gernhardt. Development and evaluation of a decompression stress index based on tissue bubble dynamics. PhD thesis, University of Pennsylvania, 1991.

[5] M.P. Hlastala and H.D. Van Liew. Absorption of in vivo inert gas bubbles. Respiration Physiology, 24(2):147-158, 1975. http://dx.doi.org/10.1016/00345687(75)90109-7.

[6] J.B. Keller. Growth and decay of gas bubbles in liquids. In R. Davies(Ed.), Proceedings of the Symposium on Cavitation in Real Liquids, pp. 20-29, New York, 1964. Elsevier.

[7] D.R. Lide. CRC Handbook of Chemistry and Physics. CRC Press, Boca Raton, Florida, 2005. 
[8] H.D. Van Liew and M.P. Hlastala. Influence of bubble size and blood perfusion on absorption of gas bubbles in tissues. Respiration Physiology, 7(1):111-121, 1969. http://dx.doi.org/10.1016/0034-5687(69)90072-3.

[9] S. Meisel, A. Nir and D. Kerem. Bubble dynamics in perfused tissue undergoing decompression. Respiration Physiology, 43(2):89-98, 1981. http://dx.doi.org/10.1016/0034-5687(81)90001-3.

[10] S.A. Mohammadein. The concentration distribution around a growing gas bubble in a bio tissue under the effect of suction process. Mathematical Biosciences, 253:88-93, 2014. http://dx.doi.org/10.1016/j.mbs.2014.04.005.

[11] S.A. Mohammadein and K.G. Mohamed. Concentration distribution around a growing gas bubble in tissue. Mathematical Biosciences, 225(1):11-17, 2010. http://dx.doi.org/10.1016/j.mbs.2010.01.003.

[12] C.M. Muth and E.S. Shank. Gas embolism. New England Journal of Medicine, 342(7):476-482, 2000. http://dx.doi.org/10.1056/NEJM200002173420706.

[13] T.S. Neuman. Arterial gas embolism and decompression sickness. News in Physiological Sciences, 17:77-81, 2002.

[14] H.B. Newton. Neurologic complications of scuba diving. Am Fam Physician, 63(11):2211-2218, 2001.

[15] V. Papadopoulou, R.J. Eckersley, C. Balestra, T.D. Karapantsios and M.-X. Tang. A critical review of physiological bubble formation in hyperbaric decompression. Advances in Colloid and Interface Science, 191-192:22-30, 2013. http://dx.doi.org/10.1016/j.cis.2013.02.002.

[16] M.S. Plesset and A. Prosperetti. Bubble dynamics and cavitation. Annual Review of Fluid Mechanics, 9:145-185, 1977. http://dx.doi.org/10.1146/annurev.fl.09.010177.001045.

[17] L.E. Scriven. On the dynamics of phase growth. Chemical Engineering Science, 10(1):1-13, 1959. http://dx.doi.org/10.1016/0009-2509(59)80019-1.

[18] R.S. Srinivasan and W.A. Gerth. Mathematical models of diffusion-limited gas bubble evolution in perfused tissue. NEDU Technical Report 13-05, Navy Experimental Diving Unit, 2013.

[19] R.S. Srinivasan, W.A. Gerth and M. R. Powell. Mathematical model of diffusionlimited evolution of multiple gas bubbles in tissue. Annals of Biomedical Engineering, 31(4):471-481, 2003. http://dx.doi.org/10.1114/1.1561288.

[20] R.S. Srinivasan, W.A. Gerth and M.R. Powell. Mathematical model of diffusionlimited gas bubble dynamics in tissue. Journal of Applied Physiology, 86(2):732$741,1999$.

[21] R.S. Srinivasan, W.A. Gerth and M.R. Powell. A mathematical model of diffusion-limited gas bubble dynamics in tissue with varying diffusion region thickness. Respiration Physiology, 123(1-2):153-164, 2000. http://dx.doi.org/10.1016/S0034-5687(00)00173-0.

[22] P. Tikuisis, K.A. Gault and R.Y. Nishi. Prediction of decompression illness using bubble models. Undersea \& Hyperbaric Medicine, 21(2):129-143, 1994.

[23] L. Van Wijngaarden. On the growth of small cavitation bubbles by convective diffusion. International Journal of Heat Mass Transfer, 10(2):127-134, 1967. http://dx.doi.org/10.1016/0017-9310(67)90093-2. 